\title{
Development of a high-resolution melting method for the screening of TNFAIP3 gene mutations
}

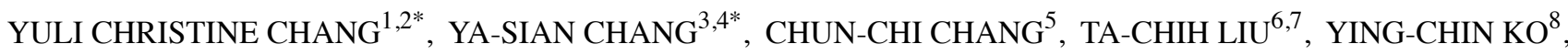 \\ CHIEN-CHIN LEE ${ }^{3}$, SHUN-JEN CHANG ${ }^{9}$ and JAN-GOWTH CHANG ${ }^{3,4,10}$ \\ ${ }^{1}$ Graduate Institute of Medicine, College of Medicine, Kaohsiung Medical University, Kaohsiung; \\ ${ }^{2}$ Department of Laboratory Medicine, Kaohsiung Medical University Hospital, Kaohsiung; \\ ${ }^{3}$ Epigenome Research Center, China Medical University Hospital, Taichung; ${ }^{4}$ Department of Laboratory Medicine, \\ China Medical University Hospital, Taichung; ${ }^{5}$ Division of Chest Medicine, Department of Internal Medicine, \\ Changhua Christian Hospital, Changhua; ${ }^{6}$ Graduate Institute of Clinical Medicine, College of Medicine, \\ Kaohsiung Medical University, Kaohsiung; ${ }^{7}$ Division of Hematology/Oncology, Department of Internal Medicine, \\ Kaohsiung Medical University Hospital, Kaohsiung; ${ }^{8}$ Environment-Omics-Disease Research Center, China Medical \\ University Hospital, Taichung; ${ }^{9}$ Department of Kinesiology, Health and Leisure Studies, National University \\ of Kaohsiung, Kaohsiung; ${ }^{10}$ School of Medicine, China Medical University, Taichung, Taiwan, R.O.C.
}

Received November 16, 2015; Accepted December 23, 2015

DOI: $10.3892 / o r .2016 .4662$

\begin{abstract}
Tumor necrosis factor, $\alpha$-induced protein 3 (TNFAIP3) which encodes a ubiquitin-modifying enzyme (A20), acts as a negative regulator of the NF- $\mathrm{B}$ pathway, and in lymphoma and autoimmune diseases it is frequently inactivated by mutations and/or deletions. We investigated the prevalence of the inactivation of TNFAIP3 in oral squamous cell carcinoma (OSCC). DNA was extracted from 81 cases of OSCC and 50 peripheral blood samples from normal controls. A high-resolution melting (HRM) analysis was used to characterize TNFAIP3 mutations, and the results were confirmed by direct DNA sequencing. Three mutations and three single-nucleotide polymorphisms (SNPs) were found to be associated with OSCC; the TNFAIP3 mutation occurred in $3.7 \%(3 / 81)$ of the OSCC cases examined. All mutations were in exon 7 [c.1081G >A (p.E361K), c.1398C >G (p.S466R) (rs200878487) and c.1760C >T (p.P587L) (rs150056192)], and p.E361K was identified as a novel mutation. We further used SIFT and PolyPhen-2 software to assess potentially functional mutations. Two SNPs, c.296-20_296-18delCTC (rs71670547) and c.380T $>$ G (p.F127C) (rs2230926), were located in exon 3, and c.2140C>T (p.P714S) was located in exon 9. A novel SNP, p.P714S differed from the one reported previ-
\end{abstract}

Correspondence to: Dr Jan-Gowth Chang, Epigenome Research Center, China Medical University Hospital, 2 Yuh-Der Road, Taichung 404, Taiwan, R.O.C.

E-mail: d6781@mail.cmuh.org.tw

"Contributed equally

Key words: high-resolution melting, direct DNA sequencing, oral squamous cell carcinoma, TNFAIP3 ously (p.P714A) (rs369155845) at that site. We also identified five SNPs in 50 normal Taiwanese individuals, and two of them [c.296-15C >T (rs377482653) and c.305A > G (p.N102S) (rs146534657)] were not found in our OSCC tissue. HRM facilitated the screening of genetic changes. In addition, our results indicate that the prevalence of the TNFAIP3 mutation is low in OSCC.

\section{Introduction}

Oral squamous cell carcinoma (OSCC) is one of the most commonly diagnosed cancers worldwide, ranking sixth among all human cancers (1). There are 650,000 new OSCC cases diagnosed and 350,000 deaths due to the disease reported each year (2). OSCC has been one of the 10 leading causes of death from cancer in Taiwan since 1991. According to the Cancer Registry Annual Report in Taiwan, oral cancer is the sixth leading cause of cancer-related death in the entire population and fourth in the male population. The development of oral cancer is highly associated with betal-quid chewing, cigarette smoking and alcohol consumption in Taiwan (3). Although technologies for diagnosis and therapy (surgery, radiation and chemotherapy) have advanced remarkably, the long-term survival rate of OSCC patients has not improved significantly for decades $(4,5)$.

The transcription factor $\mathrm{NF}-\kappa \mathrm{B}$ plays a key role in several cellular functions, including inflammation, innate and adaptive immune responses, cell proliferation, survival, angiogenesis and apoptosis. The constitutive activation of NF- $\kappa \mathrm{B}$ is common in many types of human tumors. Dysregulation of $N F-\kappa B$ promotes tumor angiogenesis and metastasis as well as resistance to chemotherapeutic agents and radiation (6). Activation of the $\mathrm{NF}-\kappa \mathrm{B}$ pathway is tightly controlled by several feedback mechanisms and is regulated by ubiquitination (7). 
Tumor necrosis factor, $\alpha$-induced protein 3 (TNFAIP3), which encodes a ubiquitin-modifying enzyme (A20), is one of the major inhibitors of the NF- $\kappa \mathrm{B}$ signaling pathway (8). It is induced by tumor necrosis factor (TNF) in human endothelial cells (9). A20 has dual functionality; it is not only able to add ubiquitin moieties to its target protein but can also cleave K63-linked polyubiquitin chains, preventing the interaction of receptor interacting serine/threonine protein kinase 1 (RIP1) and NF- $\kappa \mathrm{B}$ essential modulator (NEMO) through its deubiquitination activity mediated by its ovarian tumor (OTU) domain. A20 also adds K48-linked polyubiquitin chains to RIP1, targeting it for proteasomal degradation (10).

The TNFAIP3 gene contains eight coding exons (2-9), along with exon 1 which is non-coding, and is located on chromosome 6q23. It has been shown to be inactivated by deletions, point mutations, and/or promoter methylation in several types of lymphomas, such as B-cell lymphomas, classical Hodgkin's lymphoma, chronic lymphocytic leukemia and mucosa-associated lymphoid tissue lymphoma, which results in loss of the A20 protein (11-14). All of these lymphomas are characterized by the dysregulation of the $N F-\kappa B$ signaling pathway. These findings establish TNFAIP3 as an important tumor-suppressor gene. Human genome-wide association studies (GWAS) have linked germline single nucleotide polymorphisms of the TNFAIP3 gene with susceptibility to human inflammatory and autoimmune pathologies $(15,16)$.

The high-resolution melting (HRM) analysis is one of the most effective mutation scanning methodologies. It is a closed-tube method, such that PCR amplification and subsequent analysis are performed sequentially in the same tube, which makes it more convenient than other scanning methodologies. Moreover, there is no need for processing or separation of PCR products (17). This study aimed to assess the utility of HRM analysis using real-time polymerase chain reaction (PCR) for screening TNFAIP3 mutations.

\section{Materials and methods}

Patients and DNA extraction. A total of 81 patients who were recently diagnosed with OSCC were selected for the present study. Tissue specimens were stored immediately after resection in liquid nitrogen before DNA extraction. We included the peripheral blood samples of 50 unaffected individuals from the general population as controls. DNA was extracted as described previously by Yeh et al (18). The genomic DNA concentration was assessed using a NanoDrop 1000 spectrophotometer (NanoDrop Technologies Inc., Wilmington, DE, USA). DNA was stored at $-80^{\circ} \mathrm{C}$ until use. All tumors were classified according to the TNM classification system (19). The present study was approved by the Institutional Review Board of the China Medical University Hospital (CMUH102-REC1-015).

Design of primers for HRM assay. We used a set of primers for HRM, specific for TNFAIP3 exons 2-9 that met the requirements of the LightCycler ${ }^{\circledR} 480$ System Gene Scanning Assay. The 13 primer pairs for HRM analysis were selected using the Primer3 software (Table I). For exons 2, 7 and 9, more than one pair of primers were used to amplify the exon in two overlapping segments. All primers synthesized were of standard
Table I. Primers uses for HRM analysis of TNFAIP3 gene mutations.

\begin{tabular}{|c|c|c|}
\hline $\begin{array}{l}\text { Detection } \\
\text { for }\end{array}$ & Sequence & $\begin{array}{l}\text { Length } \\
\text { of PCR } \\
\text { amplicon } \\
\text { (bp) }\end{array}$ \\
\hline
\end{tabular}

Exon 2

P2-1 F: 5'GTCAGGCTAATAGAATGGCTTTTT 3' 250 R: 5'ATGATCTCCCGAAACTGAGGAC 3'

P2-2 F: 5'TTAAAACCATGCACCGATACACA 3' R: 5'CTATCACCCAGGCAAAAGAAACA 3'

Exon 3 F: 5'TGGGTCTTACATGCAGATAACTTG 3' 293 R: 5'CACCATGGAGCTCTGTTAGTAGAT 3'

Exon 4 F: 5'AGGGAGTACAGGATACATTCAAGC 3' 245 R: 5'AAGGCTGAAAGCATTTAAGTACAGA 3'

Exon 5 F: 5'ATGGAATTTGATGAAAGTCACCTA 3' 289 R: 5'AAGGAAAACCCTGATGTTTCAGT 3'

Exon 6 F: 5'TGAGATCTACTTACCTATGGCCTTG 3' 283 R: 5'GACACAGGAGAGAGCTGAACATAA 3'

Exon 7

P7-1 F: 5'TGTAAAATCTTGTGTGTGATTTTGTG 3' R: 5'CTCTGAGCACTCATGGCATAAAG 3'

P7-2 F: 5'CCTTCTTCATGTCTGTGAACACC 3' R: 5'CAACGTTCACAAAATCCGTTGT 3'

P7-3 F: 5'AGTGAGACCACTGCCATGAAG 3' R: 5'TTCCAGCTCTGTGGCAAGAAT 3'

P7-4 F: 5'CACCAGCGTTCCAAGTCAGAT 3' R: 5'TTCTTAAAGGTCAGGAACAAAACC 3

Exon 8 F: 5'TCTACTGTCAGCATCTCTGTATCG 3' 307 R: 5'AGCAAAAAGCATCGAACACAC 3'

Exon 9

P9-1 F: 5'AGATTTCATTGTGCTCTCCCTAAG 3' R: 5'CTGGTTGGGATGCTGACACT 3'

P9-2 F: 5'GCCTCCTGCAAGAACATCCT 3' R: 5'ATAGCACCATGATGACTGACAGC 3'

$\mathrm{F}$, forward; R, reverse.

molecular biology quality (Protech Technology Enterprise Co., Ltd., Taiwan).

HRM techniques. PCR reactions were carried out in a $10-\mu 1$ final volume using the LightCycler ${ }^{\circledR} 480$ High Resolution Melting Master (Reference 04909631001; Roche Diagnostics) and contained $1 \mathrm{X}$ buffer, Taq polymerase, nucleotides and the ResoLight dye, and $10 \mathrm{ng}$ of DNA. The primers and $\mathrm{MgCl}_{2}$ were used at $0.25 \mu \mathrm{M}$ and $2.5 \mathrm{mM}$, respectively, to detect TNFAIP3 single-nucleotide polymorphisms.

The PCR program required a SYBR Green I filter $(533 \mathrm{~nm})$, and consisted of an initial denaturation activation step at $95^{\circ} \mathrm{C}$ for $10 \mathrm{~min}$, followed by a 45 -cycle program (denaturation at $95^{\circ} \mathrm{C}$ for $10 \mathrm{sec}$, annealing at $60^{\circ} \mathrm{C}$ for $15 \mathrm{sec}$ and elongation at $72^{\circ} \mathrm{C}$ for $15 \mathrm{sec}$ with reading of the fluorescence; acquisition mode: single). The melting program included three steps: 

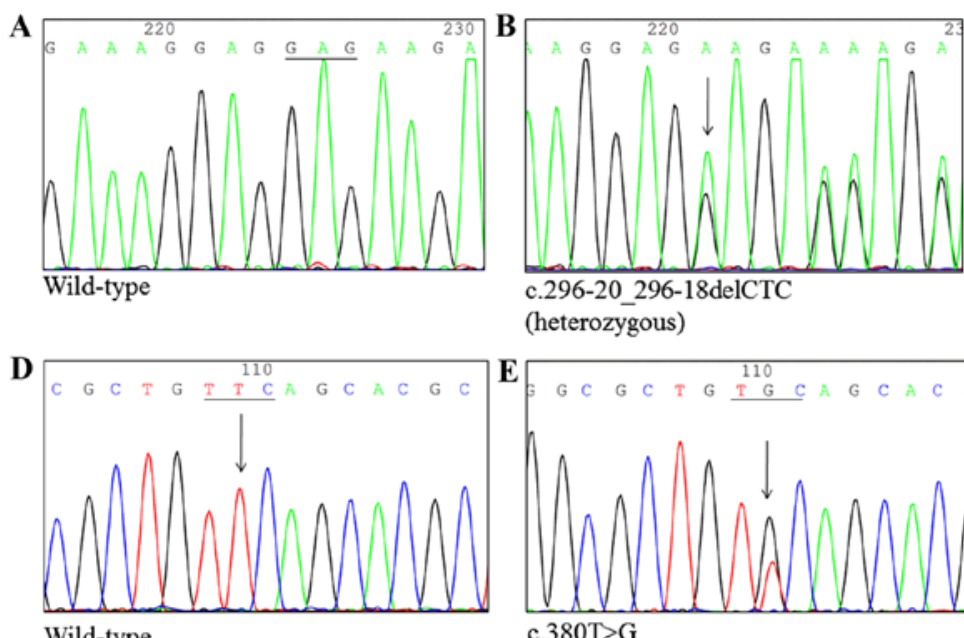

Wild-type

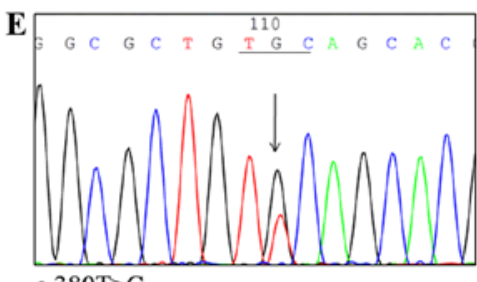

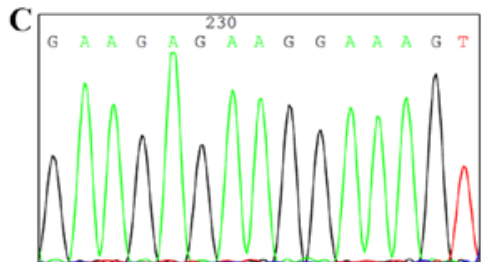

c.296-20_296-18delCTC (homozygous)

Figure 1. Direct sequencing of TNFAIP3 exon 3. Sequencing results confirmed the (A and D) wild-type and the presence of the TNFAIP3 exon 3 singlenucleotide polymorphisms: (B) c.296-20_296-18delCTC (heterozygous), (C) c.296-20_296-18delCTC (homozygous) and (E) c.380T>G.

denaturalization at $95^{\circ} \mathrm{C}$ for $1 \mathrm{~min}$, renaturation at $40^{\circ} \mathrm{C}$ for $1 \mathrm{~min}$ and then melting with a continuous reading from $60^{\circ} \mathrm{C}$ to $90^{\circ} \mathrm{C}$ at 25 acquisitions per ${ }^{\circ} \mathrm{C}$.

Direct DNA sequencing. After HRM analysis, the samples were purified using the PCR-M ${ }^{\mathrm{TM}}$ clean up system (Viogen, Sunnyvale, CA, USA). The sequence reaction was performed in a final volume of $10 \mu \mathrm{l}$, including $1 \mu \mathrm{l}$ of the purified PCR product, $2.5 \mu \mathrm{M}$ of one of the PCR primers, $2 \mu \mathrm{l}$ of the ABI PRISM terminator cycle sequencing kit v3.1 (Applied Biosystems), and $2 \mu \mathrm{l}$ of $5 \mathrm{X}$ sequencing buffer. The sequencing program followed a 25 -cycle PCR program (denaturation at $96^{\circ} \mathrm{C}$ for $10 \mathrm{sec}$, annealing at $50^{\circ} \mathrm{C}$ for $5 \mathrm{sec}$, and elongation at $60^{\circ} \mathrm{C}$ for $\left.4 \mathrm{~min}\right)$. Sequence detection was performed using an ABI Prism 3130 Genetic Analyzer (Applied Biosystems) according to standard protocols.

Statistical analysis. Data analysis was performed using the SPSS version 17.0 software (SPSS, Inc., Chicago, IL, USA). The Chi-square test was used to compare TNFAIP3 genotype distributions between the case and control groups. Univariate unconditional logistic regression analyses were used to obtain odds ratios (OR) and corresponding 95\% confidence intervals (CI). A value of $\mathrm{P}<0.05$ was considered to indicate a statistically significant difference.

\section{Results}

Screening of TNFAIP3 mutations in exons 2, 4, 5, 6 and 8. To identify TNFAIP3 gene mutations in exons 2, 4, 5, 6 and 8, we designed primer pairs specific for each exon region (Table I). HRM analysis of exons 2, 4, 5, 6 and 8 showed no nucleotide changes in these regions (data not shown).

Screening of TNFAIP3 mutations in exon 3. We used one primer pair to identify TNFAIP3 mutations in exon 3 . The PCR amplification product contained multiple SNPs. Thus, this PCR primer was not suitable for HRM. Consequently, this exon was analyzed by direct sequencing. Fig. 1 shows that one SNP resulted in a change from thymine to guanine at posi- tion 380 (c.380T>G), and another caused a deletion of cytosine, thymine and cytosine (CTC) at position 296 (c.296-20_29618 delCTC). The c.380T $>\mathrm{G}$ change resulted in a Phe127Cys amino acid substitution. In addition, the c.380 $>\mathrm{G}$ nucleotide change was found in four subjects, for an incidence of $4.94 \%$, while a three nucleotide deletion (c.296-20_296-18delCTC) was found in 80 subjects, for the highest incidence of $98.77 \%$.

Screening of TNFAIP3 mutations in exon 7 . We used four primer pairs (P7-1, P7-2, P7-3 and P7-4) to identify TNFAIP3 mutations in exon 7. As shown in Fig. 2, three mutations resulted in a change from guanine to adenine at position 1081 (c.1081G>A), from cytosine to guanine at position 1398 (c.1398C $>$ G), and from cytosine to thymine at position $1760(\mathrm{c} .1760 \mathrm{C}>\mathrm{T})$. These were confirmed by direct sequencing of the codons of interest. The c.1081G $>$ A, c.1398C $>\mathrm{G}$ and c.1760C $>$ T changes resulted in Glu361Lys, Ser466Arg, and Pro587Leu amino acid substitutions, respectively. Intriguingly, the mutation p.E361K has not been reported previously, which the PolyPhen-2 predicted as a benign mutation. In addition, mutations in exon 7 were found in three subjects, for an incidence of $3.7 \%$.

Screening of TNFAIP3 mutations in exon 9. We used two primer pairs (P9-1 and P9-2) to identify TNFAIP3 mutations in exon 9. One SNP resulted in a change from cytosine to thymine at position 2140 (c.2140C $>$ T) (Fig. 3). This was confirmed by direct sequencing. The c. $2140 \mathrm{C}>\mathrm{T}$ change resulted in a Pro714Ser amino acid substitution. Intriguingly, the SNP p.P714S is similar to the amino acid change Pro714Ala, which has been reported previously (rs369155845). In addition, a c. $2140 \mathrm{C}>\mathrm{T}$ nucleotide change was found in one subject, for an incidence of $1.2 \%$.

TNFAIP3 polymorphisms in normal controls. We recruited 50 healthy Taiwanese subjects from the China Medical University Hospital. DNA from the subjects was screened for TNFAIP3 mutations. We identified five SNPs by HRM analysis, which were confirmed by direct DNA sequencing: c.296-20_296-18delCTC, c.296-15C>T, c.305A >G, c.380T>G and c. $2140 \mathrm{C}>\mathrm{T}$ (Fig. 4). In addition, the nucleotide changes 

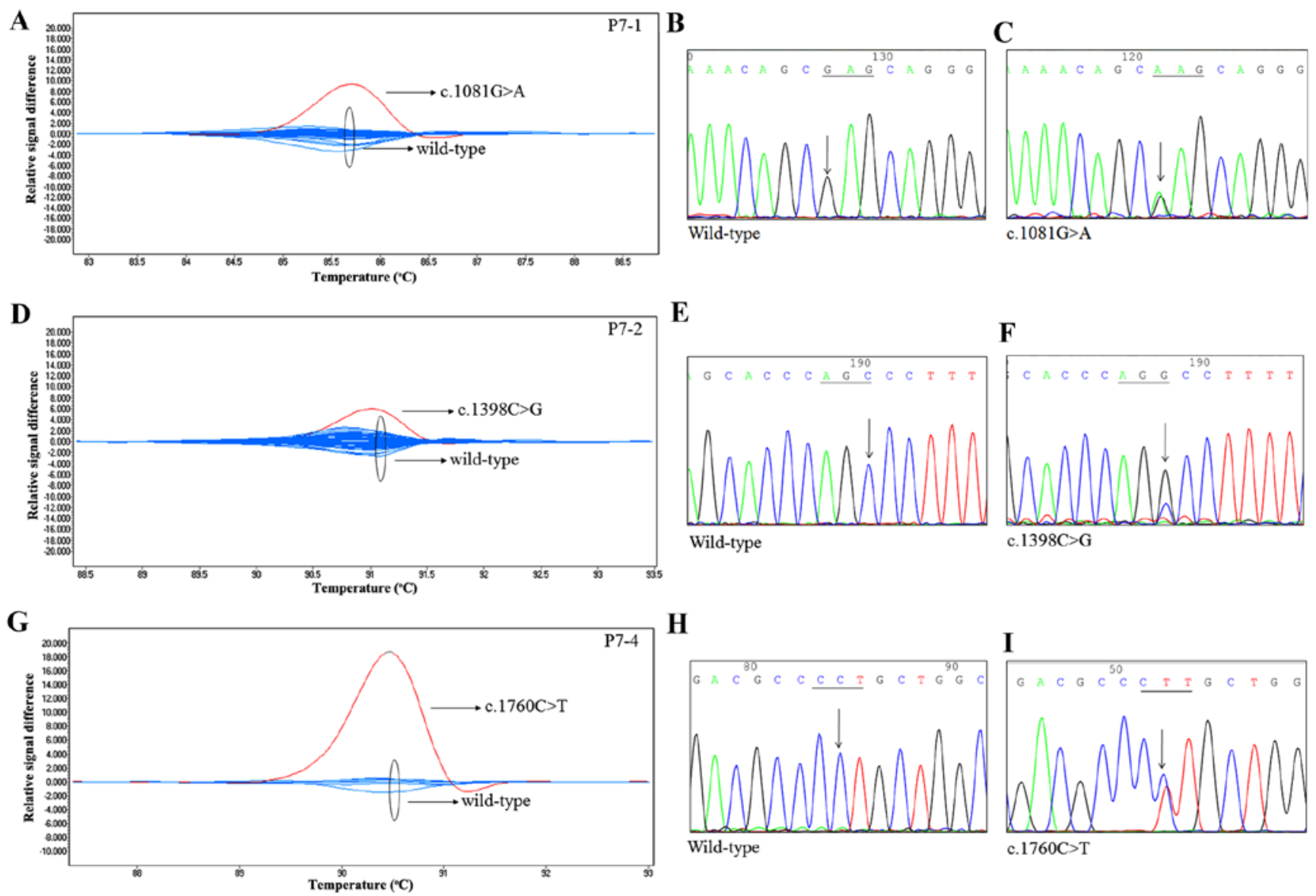

Figure 2. HRM assays for TNFAIP3 in exon 7. (A) Mutation screening at exon 7 of the TNFAIP3 gene using the primer pair P7-1. Sequencing results confirmed the (B) wild-type and the presence of the TNFAIP3 exon 7 mutation (C) c.1081G $>$ A. (D) Mutation screening at exon 7 of the TNFAIP3 gene using primer pairs P7-2. Sequencing confirmed the (E) wild-type and the presence of the TNFAIP3 exon 7 mutation (F) c.1398C $>$ G. (G) Mutation screening at exon 7 of the TNFAIP3 gene using the primer pair P7-4. Sequencing results confirmed the (H) wild-type and the presence of the TNFAIP3 exon 7 mutation (I) c.1760C $>$ T.

Table II. Haplotype frequency of TNFAIP3_rs71670547, rs377482653, rs146534657 and rs2230926 among the OSCC patients.

\begin{tabular}{lccccc}
\hline Haplotype & $\begin{array}{c}\text { Cases } \\
\mathrm{n}(\%)\end{array}$ & $\begin{array}{c}\text { Controls } \\
\mathrm{n}(\%)\end{array}$ & OR & $95 \%$ CI & P-value \\
\hline del-C-A-T & $149(92)$ & $88(88)$ & 1 & Ref & \\
CTC-C-A-G & $4(2)$ & $7(7)$ & 0.34 & $0.10-1.19$ & 0.0771 \\
CTC-C-A-T & $9(6)$ & $3(3)$ & 1.77 & $0.47-6.72$ & 0.3946 \\
CTC-C-G-T & $0(0)$ & $1(1)$ & & & \\
del-T-A-T & $0(0)$ & $1(1)$ & & & \\
\hline
\end{tabular}

OR, odds ratio; CI, confidence interval.

with c.296-20_296-18delCTC, c.296-15C>T, c.305A>G, c. $380 \mathrm{~T}>\mathrm{G}$ and c. $2140 \mathrm{C}>\mathrm{T}$ were found in $50,1,1,7$, and 1 subject, respectively, for incidences of 100, 2, 2, 14 and 2\%, respectively.

Frequencies of the TNFAIP3 haplotype. The rs71670547, rs377482653, rs146534657 and rs2230926 haplotypes were analyzed to investigate susceptibility to OSCC. The haplotype CTC-C-A-G was marginally inversely associated with a high risk of $\mathrm{OSCC}(\mathrm{OR}=0.34 ; 95 \% \mathrm{CI}=0.10-1.19 ; \mathrm{P}=0.077$; Table II $)$.

\section{Discussion}

Several TNFAIP3 mutations involving various substitutions in multiple tumor types have been reported [Table III, extracted from the Catalogue of Somatic Mutations in the Cancer (COSMIC) Database]. Many studies have focused on TNFAIP3 genetic variations in hematopoietic and lymphoid tissue. According to the COSMIC database, the TNFAIP3 mutation in OSCC has been reported by only one study from the USA using whole-exome sequencing (20), and the only mutation (p.Y614C) identified in that study was not found in the Taiwanese population of our study. Conversely, we identified three mutations by HRM analysis (p.E361K, p.S466R and p.P587L) and confirmed them by direct sequencing. This discrepancy in results may reflect different lifestyle risk factors. For example, oral cancer patients from Taiwan are frequently exposed to betel-quid chewing, and the betel-quid used in Taiwan is different from that used in other countries.

Although the HRM method is a powerful screening tool, it has some limitations; one of which is that unexpected polymorphisms present in the mutation of interest may interfere with genotyping. Therefore, we designed amplicon lengths of 100-300 bp, the suggested ideal size for HRM analysis. Two polymorphisms in intron 2 (rs71670547 and rs377482653) and two in exon 3 (rs146534657 and rs2230926) caused difficul- 


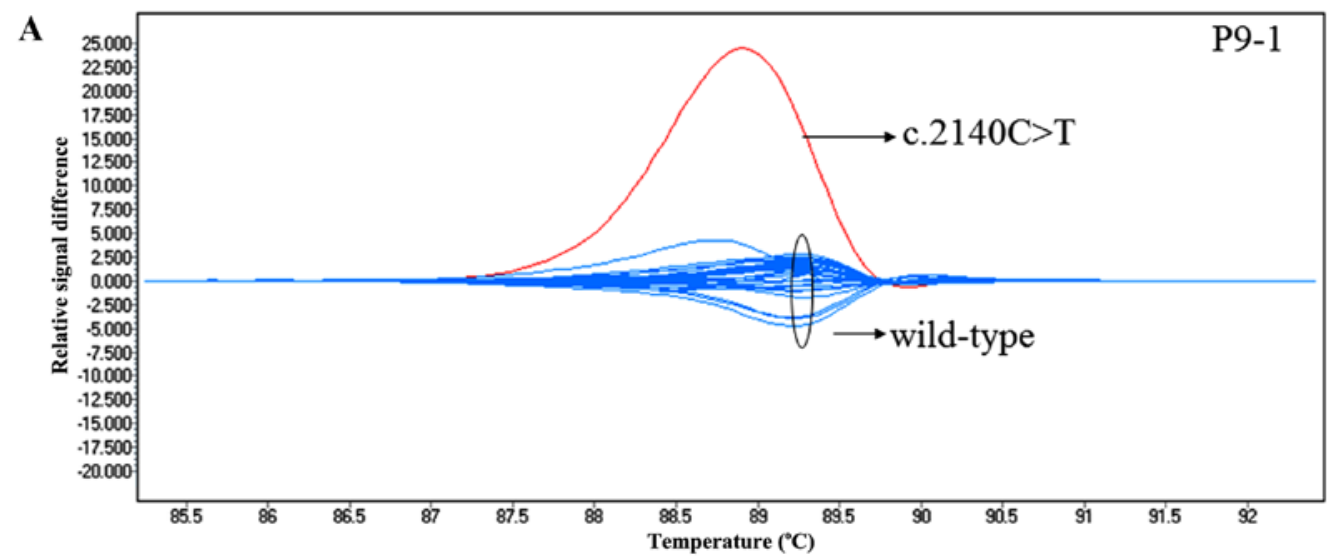

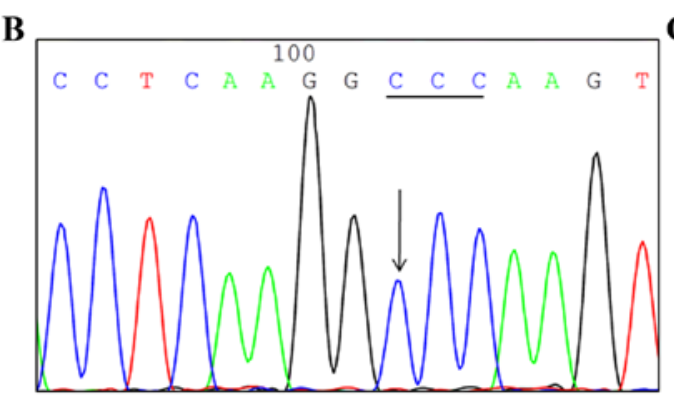

Wild-type

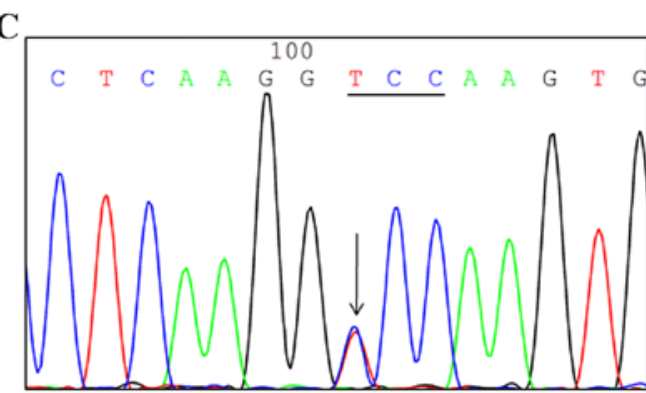

c. $2140 \mathrm{C}>\mathrm{T}$

Figure 3. HRM assays for TNFAIP3 exon 9. (A) Normalized and temperature-shifted difference plots showing two melting profiles, the wild-type (WT) sample is blue, and the single nucleotide alteration is red. Sequencing results confirmed the (B) wild-type and the presence of the TNFAIP3 exon 9 singlenucleotide polymorphism (C) c.2140C $>\mathrm{T}$.

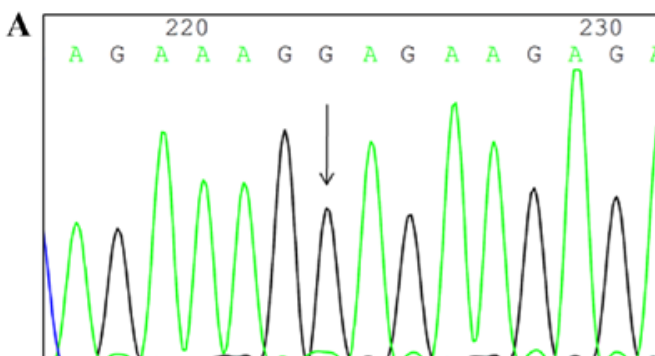

Wild-type

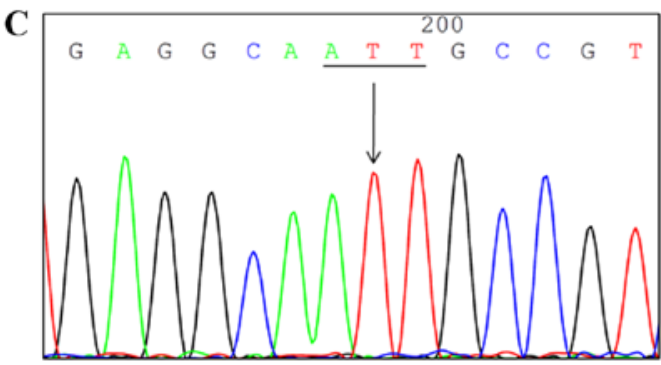

Wild-type

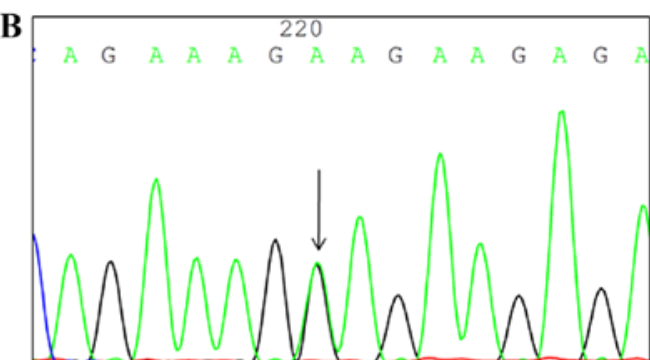

c. $296-15 \mathrm{C}>\mathrm{T}$

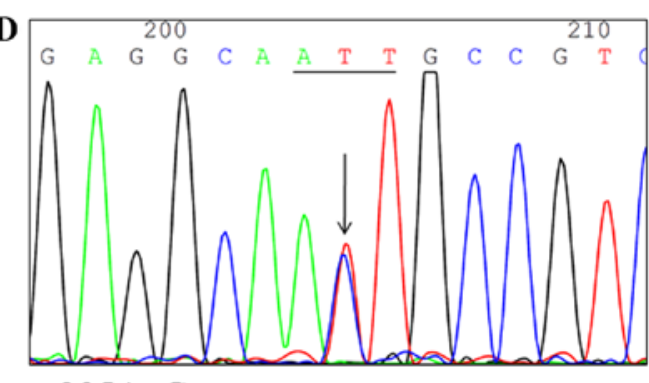

c. $305 \mathrm{~A}>\mathrm{G}$

Figure 4. Application of HRM and direct sequencing for analysis of TNFAIP3 single-nucleotide polymorphisms in 50 normal individuals. Sequencing results confirmed the (A and C) wild-type and the presence of the TNFAIP3 exon 3 single-nucleotide polymorphisms (B) c.296-15C $>$ T, and (D) c.305A>G.

ties in the HRM analysis; these polymorphisms could not be differentiated using a melting curve. To avoid this, the designed primers should flank the exon or intron as closely as possible. When an SNP is close to the exon or intron boundary, the primer can be placed over the SNP and a mismatched base with no allelic preference can be introduced at the SNP position (21). If the amplicon length is increased, the wild-type and heterozygote curves become smaller and are more difficult to distinguish. The HRM method is unable to detect mutations encompassing an entire exon or deletions of entire genes and exons. 


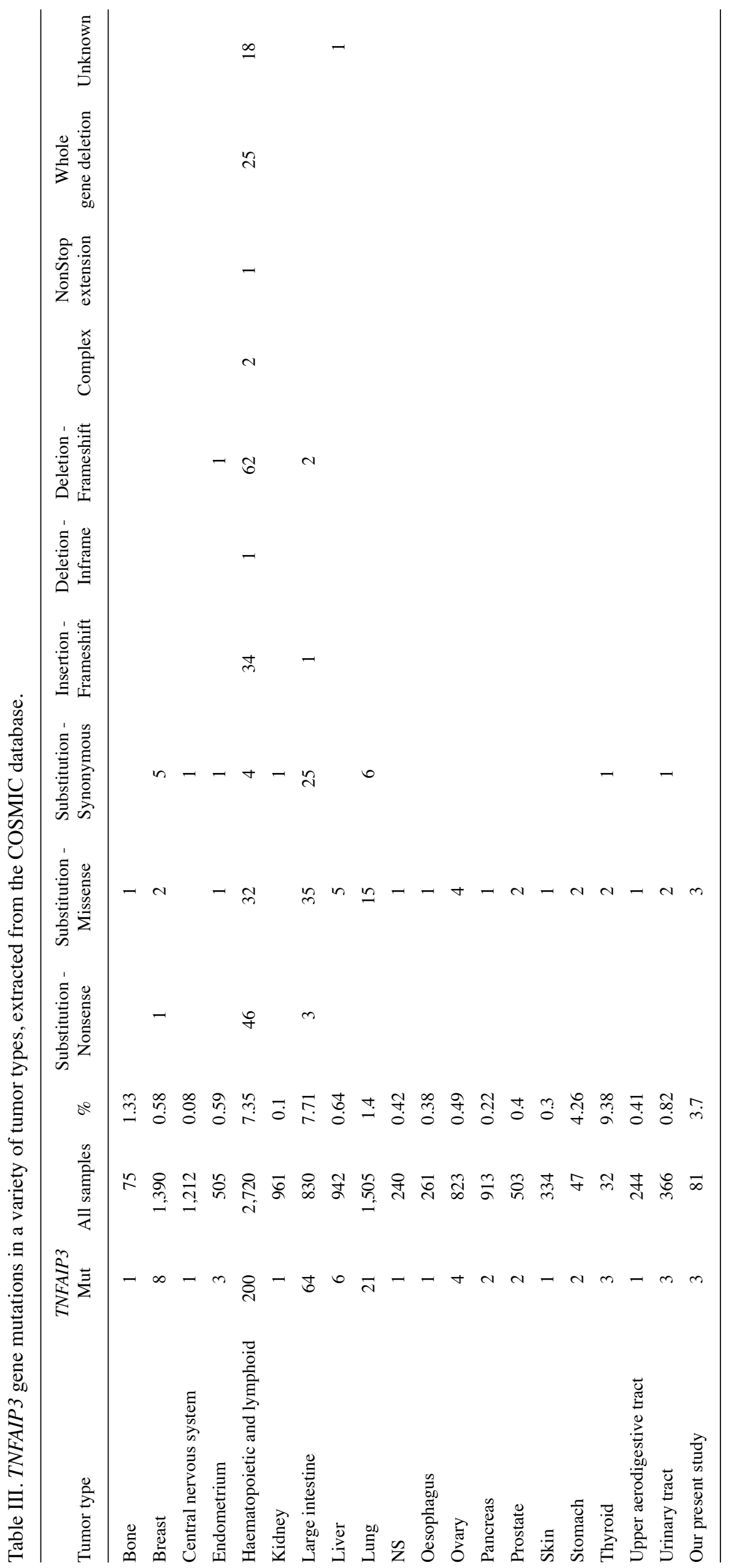


Mice with the A20 deletion in intestinal epithelial cells (IECs), B cells, myeloid cells, dendritic cells (DCs) and keratinocytes have been investigated extensively. Specific A20 deletions in B cells exhibit enhanced B-cell proliferation and survival and autoantibody production (22-24). Mice with the A20 deletion in all cells of myeloid origin develop spontaneous polyarthritis with the production of type II collagen autoantibodies and inflammatory cytokines in serum (25). In addition, mice with a DC-specific A20 deletion developed either SLE-like symptoms or human inflammatory bowel disease in independent studies $(26,27)$. Furthermore, mice with A20-deficient IECs are highly sensitive to dextran sodium sulfate-induced colitis and TNF due to IEC apoptosis and loss of barrier integrity (28). Finally, A20 expression was significantly decreased in human colorectal cancer samples compared with adjacent non-neoplastic mucosa (29), and mice with A20-deficient keratinocytes displayed keratinocyte hyperproliferation and ectodermal organ abnormalities (30). Thus, conditional gene targeting studies have demonstrated an important role for A20 in controlling tissue homeostasis.

In conclusion, the HRM DNA screening method provides a reliable, accurate, and rapid method of identifying TNFAIP3 mutations for the clinical diagnosis of cancer.

\section{Acknowledgements}

This research was supported by grants from the Taiwan Ministry of Health and Welfare Clinical Trial and Research Center of Excellence (MOHW105-TDU-B-212-133019 and MOHW104-TDU-B-212-124-002) and China Medical University Hospital (DMR-103-121 and DMR-103-116).

\section{References}

1. Molinolo AA, Amornphimoltham P, Squarize $\mathrm{CH}$, Castilho RM, Patel V and Gutkind JS: Dysregulated molecular networks in head and neck carcinogenesis. Oral Oncol 45: 324-334, 2009.

2. Leemans CR, Braakhuis BJ and Brakenhoff RH: The molecular biology of head and neck cancer. Nat Rev Cancer 11: 9-22, 2011.

3. Ko YC, Huang YL, Lee CH, Chen MJ, Lin LM and Tsai CC: Betel quid chewing, cigarette smoking and alcohol consumption related to oral cancer in Taiwan. J Oral Pathol Med 24: 450-453, 1995.

4. Tanaka T, Tanaka M and Tanaka T: Oral carcinogenesis and oral cancer chemoprevention: A review. Pathol Res Int 2011: 431246 2011.

5. Petersen PE: Oral cancer prevention and control - the approach of the World Health Organization. Oral Oncol 45: 454-460, 2009.

6. Karin M and Greten FR: NF-kappaB: Linking inflammation and immunity to cancer development and progression. Nat Rev Immunol 5: 749-759, 2005

7. Oeckinghaus A, Hayden MS and Ghosh S: Crosstalk in NF-кB signaling pathways. Nat Immunol 12: 695-708, 2011.

8. Catrysse L, Vereecke L, Beyaert R and van Loo G: A20 in inflammation and autoimmunity. Trends Immunol 35: 22-31, 2014.

9. Dixit VM, Green S, Sarma V, Holzman LB, Wolf FW, O'Rourke K, Ward PA, Prochownik EV and Marks RM: Tumor necrosis factor-alpha induction of novel gene products in human endothelial cells including a macrophage-specific chemotaxin. J Biol Chem 265: 2973-2978, 1990.

10. Wertz IE, O'Rourke KM, Zhou H, Eby M, Aravind L, Seshagiri S, Wu P, Wiesmann C, Baker R, Boone DL, et al: De-ubiquitination and ubiquitin ligase domains of A20 downregulate NF-kappaB signalling. Nature 430: 694-699, 2004.

11. Kato M, Sanada M, Kato I, Sato Y, Takita J, Takeuchi K, Niwa A, Chen Y, Nakazaki K, Nomoto J, et al: Frequent inactivation of A20 in B-cell lymphomas. Nature 459: 712-716, 2009.
12. Nomoto J, Hiramoto N, Kato M, Sanada M, Maeshima AM, Taniguchi H, Hosoda F, Asakura Y, Munakata W, Sekiguchi N, et al: Deletion of the TNFAIP3/A20 gene detected by FICTION analysis in classical Hodgkin lymphoma. BMC Cancer 12: 457 , 2012.

13. Philipp C, Edelmann J, Bühler A, Winkler D, Stilgenbauer S and Küppers R: Mutation analysis of the TNFAIP3 (A20) tumor suppressor gene in CLL. Int J Cancer 128: 1747-1750, 2011.

14. Chanudet E, Huang Y, Ichimura K, Dong G, Hamoudi RA, Radford J, Wotherspoon AC, Isaacson PG, Ferry J and Du MQ: A20 is targeted by promoter methylation, deletion and inactivating mutation in MALT lymphoma. Leukemia 24: 483-487, 2010.

15. Ma A and Malynn BA: A20: Linking a complex regulator of ubiquitylation to immunity and human disease. Nat Rev Immunol 12: 774-785, 2012.

16. Graham RR, Hom G, Ortmann W and Behrens TW: Review of recent genome-wide association scans in lupus. J Intern Med 265: 680-688, 2009

17. Er TK and Chang JG: High-resolution melting: Applications in genetic disorders. Clin Chim Acta 414: 197-201, 2012.

18. Yeh KT, Shih MC, Lin TH, Chen JC, Chang JY, Kao CF, Lin KL and Chang JG: The correlation between $\mathrm{CpG}$ methylation on promoter and protein expression of E-cadherin in oral squamous cell carcinoma. Anticancer Res 22: 3971-3975, 2002.

19. Sobin LH and Fleming ID: TNM Classification of Malignant Tumors, 5th edition (1997). Union Internationale Contre le Cancer and the American Joint Committee on Cancer. Cancer 80: 1803-1804, 1997.

20. Stransky N, Egloff AM, Tward AD, Kostic AD, Cibulskis K, Sivachenko A, Kryukov GV, Lawrence MS, Sougnez C, McKenna A, et al: The mutational landscape of head and neck squamous cell carcinoma. Science 333: 1157-1160, 2011.

21. Shih HC, Er TK, Chang TJ, Chang YS, Liu TC and Chang JG: Rapid identification of HBB gene mutations by high-resolution melting analysis. Clin Biochem 42: 1667-1676, 2009.

22. Tavares RM, Turer EE, Liu CL, Advincula R, Scapini P, Rhee L, Barrera J, Lowell CA, Utz PJ, Malynn BA, et al: The ubiquitin modifying enzyme A20 restricts B cell survival and prevents autoimmunity. Immunity 33: 181-191, 2010.

23. Hövelmeyer N, Reissig S, Xuan NT, Adams-Quack P, Lukas D, Nikolaev A, Schlüter D and Waisman A: A20 deficiency in B cells enhances B-cell proliferation and results in the development of autoantibodies. Eur J Immunol 41: 595-601, 2011.

24. Chu Y, Vahl JC, Kumar D, Heger K, Bertossi A, Wójtowicz E, Soberon V, Schenten D, Mack B, Reutelshöfer M, et al: B cells lacking the tumor suppressor TNFAIP3/A20 display impaired differentiation and hyperactivation and cause inflammation and autoimmunity in aged mice. Blood 117: 2227-2236, 2011.

25. Matmati M, Jacques P, Maelfait J, Verheugen E, Kool M, Sze M, Geboes L, Louagie E, Mc Guire C, Vereecke L, et al: A20 (TNFAIP3) deficiency in myeloid cells triggers erosive polyarthritis resembling rheumatoid arthritis. Nat Genet 43: 908-912, 2011.

26. Kool M, van Loo G, Waelput W, De Prijck S, Muskens F, Sze M, van Praet J, Branco-Madeira F, Janssens S, Reizis B, et al: The ubiquitin-editing protein A20 prevents dendritic cell activation, recognition of apoptotic cells, and systemic autoimmunity. Immunity 35: 82-96, 2011.

27. Hammer GE, Turer EE, Taylor KE, Fang CJ, Advincula R, Oshima S, Barrera J, Huang EJ, Hou B, Malynn BA, et al: Expression of A20 by dendritic cells preserves immune homeostasis and prevents colitis and spondyloarthritis. Nat Immunol 12: 1184-1193, 2011.

28. Vereecke L, Sze M, Mc Guire C, Rogiers B, Chu Y, Schmidt-Supprian M, Pasparakis M, Beyaert R and van Loo G: Enterocyte-specific A20 deficiency sensitizes to tumor necrosis factor-induced toxicity and experimental colitis. J Exp Med 207: 1513-1523, 2010.

29. Ungerbäck J, Belenki D, Jawad ul-Hassan A, Fredrikson M, FransénK,ElanderN, VermaD and SöderkvistP: Genetic variation and alterations of genes involved in NFKB/TNFAIP3- and NLRP3-inflammasome signaling affect susceptibility and outcome of colorectal cancer. Carcinogenesis 33: 2126-2134, 2012.

30. Lippens S, Lefebvre S, Gilbert B, Sze M, Devos M, Verhelst K, Vereecke L, Mc Guire C, Guérin C, Vandenabeele P, et al: Keratinocyte-specific ablation of the NF- $\kappa \mathrm{B}$ regulatory protein A20 (TNFAIP3) reveals a role in the control of epidermal homeostasis. Cell Death Differ 18: 1845-1853, 2011. 